\title{
Surgical Treatment of Synchronous Gastric and Esophageal Carcinoma: Case Report and Review of Literature
}

\author{
Songping Xie ${ }^{1}$ jie Huang ${ }^{1}$ Ganjun Kang ${ }^{1}$ Guohua Fan ${ }^{1}$ Wei Wang ${ }^{1}$ \\ ${ }^{1}$ Department of Thoracic Surgery, Renmin Hospital, Wuhan \\ University, Wuhan, Hubei, China \\ Address for correspondence Jie Huang, MD, Department of Thoracic \\ Surgery, Renmin Hospital, Wuhan University, Wuhan, Hubei Province \\ 430060, China (e-mail: doctor_huangjie@126.com). \\ Thorac Cardiovasc Surg Rep 2013;2:35-37.
}

\begin{abstract}
Keywords

- esophageal cancer

- gastric cancer

- synchronous

- surgery

A 65-year-old man was admitted to our hospital because of advanced esophageal squamous cell carcinoma located on the left posterior wall of the lower thoracic esophagus and gastric adenocarcinoma in the antrum. Esophagectomy and distal gastrectomy with two-field lymph node dissection (mediastinum and abdomen) were performed via a left-sided abdominothoracic incision. The remnant gastric was pulled up successfully with the blood supply maintained by the left gastric vessel. He was discharged on the 13th postoperative day without any complications.
\end{abstract}

\section{Introduction}

A 65-year-old man with progressive dysphagia for 2 months was admitted to our hospital in December 2012. His medical history was notable for alcohol abuse for 40 years. Physical examination of the chest and abdomen revealed no abnormal findings. Upper gastrointestinal endoscopic examination showed double cancers with thoracic esophageal cancer, located on the left posterior wall of the lower thoracic esophagus, 30 to $37 \mathrm{~cm}$ from the incisors. Biopsy of the esophageal mass revealed squamous cell carcinoma, and gastric cancer in the antrum, distal part of stomach, with the pathological analysis revealed adenocarcinoma. Computed tomography (CT) of the chest and abdomen showed no distant or lymph node metastases, showed an irregular wall thickening at the lower esophagus (-Fig. 1), and revealed a heterogeneous mass in the region of the antrum of stomach. Barium esophagography revealed an approximately $6 \mathrm{~cm}$ arc filling defect, local mucosal damages and wall stiffness, irregularities in lower thoracic esophagus (-Fig. 2), and $1 \mathrm{~cm}$ arc filling defect in distal of stomach with intervening normal mucosa(-Fig. 3). Clinical staging of the double cancer was stage II (T3NOMO) in esophageal cancer and stage IB (T2NOMO) in gastric cancer. received

April 30, 2013

accepted after revision

June 24, 2013

published online

August 29, 2013
Before surgery, we planned to perform total gastrectomy and esophageal resection by using colon for reconstruction. The bowel was prepared by mechanical cleansing and examined completely by endoscope. He underwent radical enbloc esophagectomy and gastrectomy via a left-sided abdominothoracic incision through the seventh intercostal space, with a two-field lymph node dissection in the upper abdomen and mediastinum. The upper end of the esophagus was resected $5 \mathrm{~cm}$ above the tumor. The right and left gastroepiploic vessels, and the right gastric vessels were divided; only the left gastric vessels were preserved. Because the gross appearance of the stomach seemed to be normal, we decided to preserve the remnant stomach and perform distal gastrostomy. The distal gastrectomy was performed with preservation of half of the stomach as a minimum requirement, with a $10-\mathrm{cm}$ distance from the tumor to the closest resection line. A gastric tube was extended far enough to reach the proximal esophagus. The esophagogastric anastomosis was performed mechanically under the level of the left inferior pulmonary vein, with a circular stapler by an anterior gastrotomy to the high point of the fundus of the gastric. The jejunum was lifted through the retrocolic route, an end-to-side gastrojejunostomy was made mechanically approximately $30 \mathrm{~cm}$ distal to the ligament of Treitz ( $\mathbf{- F i g s .} 4$ and $\mathbf{5}$ ), and Braun anastomosis was performed approximately $20 \mathrm{~cm}$ distal to the gastrojejunostomy. No
License terms Stuttgart · New York
DOI http://dx.doi.org/

10.1055/s-0033-1351357. ISSN 2194-7635. 


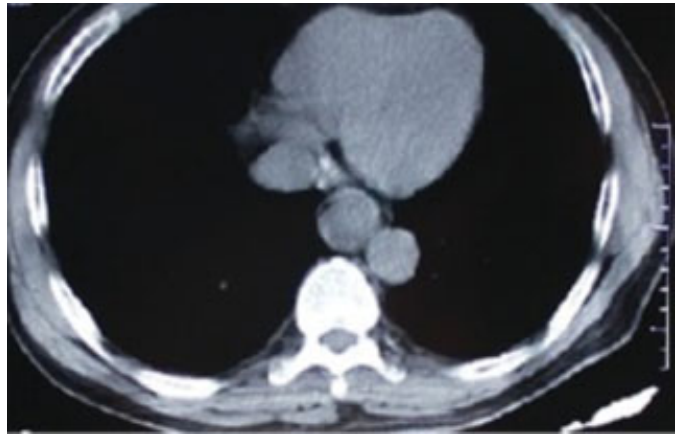

Fig. 1 Computed tomography of the chest showed an irregular wall thickening at the lower esophagus.

micro vascular anastomosis was performed. The remnant stomach appeared rosy with adequate blood supply by the left gastric vessels. Enteral nutrition therapy was made by stoma of jejunum. The operating time was 239 minutes and the estimated blood loss was $380 \mathrm{~mL}$. Postoperative course was uneventful. The integrity of the anastomosis of the esophagogastrostomy was confirmed by water soluble contrast radiography on postoperative day 9 , and there was no sign of leakage or stricture. He started a liquid diet the following day and he was discharged on the 13th postoperative day without any major postoperative complications. Histologically, the esophageal tumor was diagnosed as middle differentiated squamous cell carcinoma with tumor-free margins. The gastric tumor was diagnosed as poorly differentiated adenocarcinoma with tumor-free margins. Metastasis was not found in the abdomen and mediastinum lymph node.

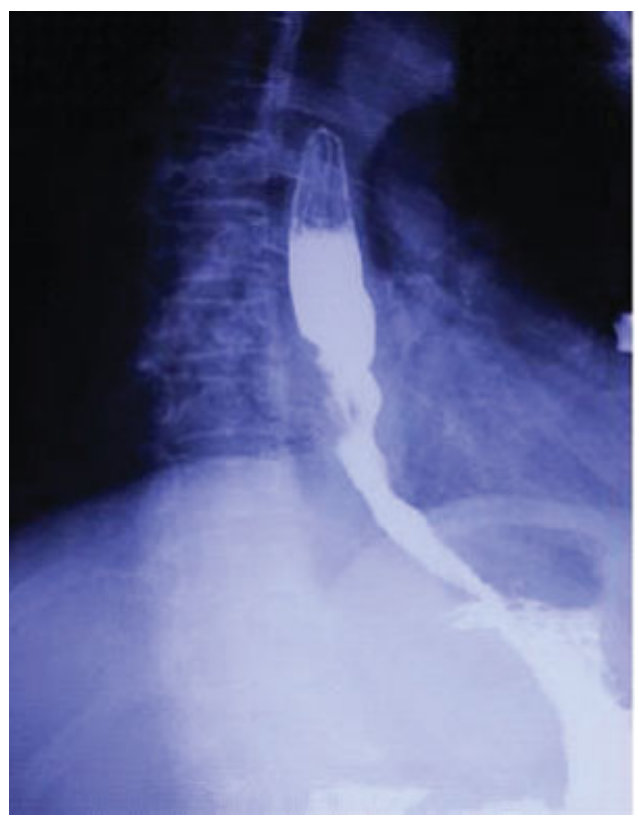

Fig. 2 Barium esophagography revealed that one-third of the lower esophagus showed an approximately $6 \mathrm{~cm}$ arc filling defect, local mucosal damages and wall stiffness, and irregularities in lower thoracic esophagus.

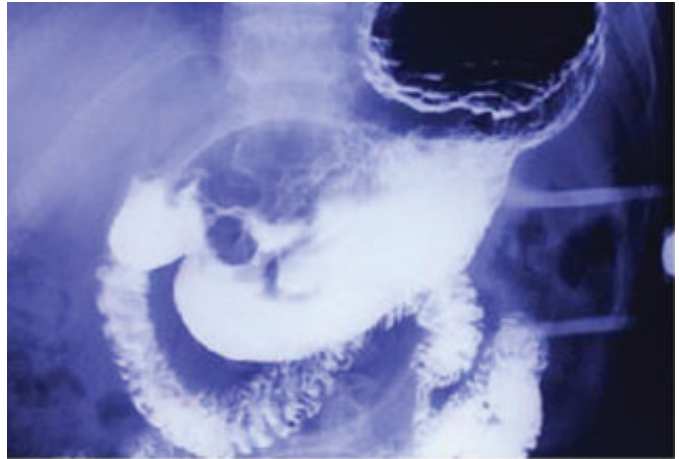

Fig. 3 Barium esophagography revealed $1 \mathrm{~cm}$ arc filling defect in distal of stomach with intervening normal mucosa.

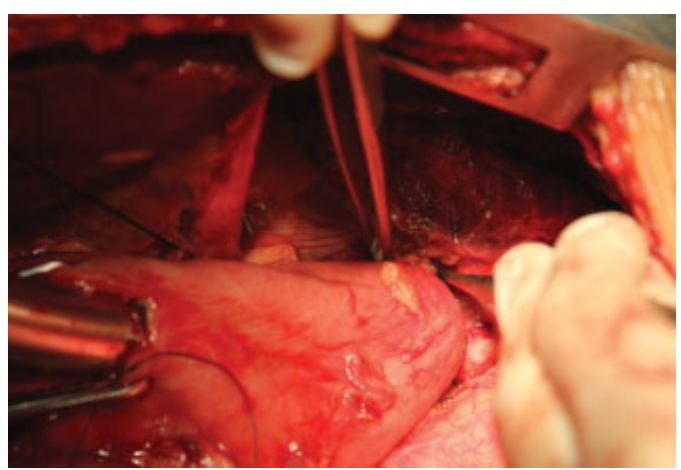

Fig. 4 The esophagogastric anastomosis was performed mechanically under the level of the left inferior pulmonary vein.

The frequency of synchronous esophageal and gastric carcinomas occurs most frequently due to the development of more sophisticated invasive and noninvasive diagnostic tools and an increase in the number of elderly patients. ${ }^{1}$ However, sometimes the obstruction caused by esophageal cancer impacts the movement of gastroscope into the stomach, so it is likely to cause a missed diagnosis of gastric cancer. Therefore, the presence of another cancer should be carefully observed in esophageal cancer. For patients with severe esophageal obstruction, the gastroscope cannot enter into the stomach, examinations such as upper gastrointestinal barium meal and chest and abdominal CT should be used to

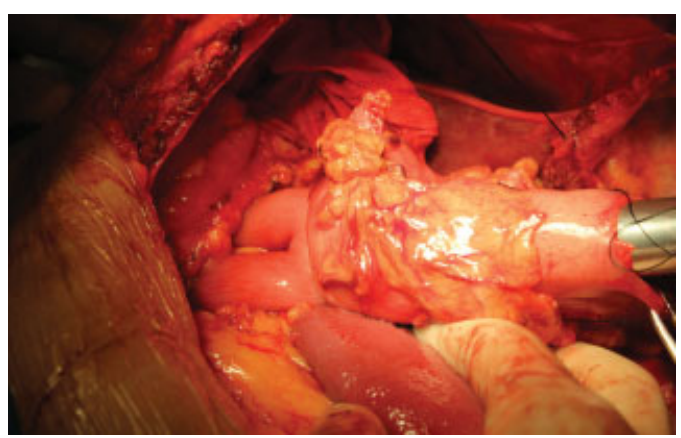

Fig. 5 An end-to-side gastrojejunostomy was made mechanically approximately $30 \mathrm{~cm}$ distal to the ligament of Treitz. 
understand the existence of gastric cancer. Positron emission tomography-CT is useful for detecting unknown synchronous esophageal and gastric carcinomas.

Koide et $\mathrm{al}^{2}$ reported that outcome of cases with synchronous gastric tumors associated with esophageal cancer is not poor compared with that of the nonassociated cases. If early detected and properly treated, these patients would achieve acceptable survival. The key of the surgical treatment of esophageal and gastric cancer in the same period lies in the reconstruction of the digestive tract. Total gastrectomy is more invasive because it needs reconstruction with another organ such as the colon. Therefore, it is important to detect the lesion so as to treat it with minimally invasive surgery such as partial resection. The operation should be conducted according to tumor location and stomach conditions. If stomach left enough for alimentary tract restoration after the esophagus tumor resection, the remnant stomach will be selected to replace the esophagus. Therefore, postoperative complications will show a lower incidence, and the hospital stay will be shorter. If the stomach cannot be used, then alimentary tract restoration can be accomplished using the colon.

\section{References}

1 Pasławski M, Złomaniec J, Rucińska E, Kołtyś W. Synchronous primary esophageal and gastric cancers. Ann Univ Mariae Curie Sklodowska Med 2004;59(1):406-410

2 Koide N, Adachi W, Koike S, Watanabe H, Yazawa K, Amano J. Synchronous gastric tumors associated with esophageal cancer: a retrospective study of twenty-four patients. Am J Gastroenterol 1998;93(5):758-762 